\title{
Estimation of above ground biomass of oil palm trees by PALSAR
}

\begin{abstract}
The main objective of this paper is to show the potential use of L-Band SAR polarimetry. We used SAR images to estimate the above ground biomass (AGB) of an oil palm plantation. The approach used in this study was to analyze the relationship between the radar backscatter and AGB data by a specific allometric equation in variation of age. The four polarization of PALSAR was adapted in this study and converted to sigma nought for respective ages. Laplacian filter with window size $3 \times 3,5 \times 5$ and $7 \times 7$ was used on the PALSAR polarimetry images. Laplacian filter was used to show the best improvement to the relation of radar backscattering and AGB of oil palm trees. As a conclusion, improved radar backscattering of oil palm trees can be useful to estimate AGB of oil palm trees.
\end{abstract}

Keyword: Above ground biomass; PALSAR; Oil palm plantation 